Revista Brasil. Bot., V.31, n.4, p.611-620, out.-dez. 2008

\title{
Identificação de flavonóides em Hypericum cordatum (Vell.) N. Robson (Clusiaceae) ${ }^{1}$
}

\author{
RODRIGO STROHMAYER DOURADO² e ÂNGELA MARIA LADEIRA',3
}

(recebido: 16 de março de 2007; aceito: 29 de agosto de 2008)

\begin{abstract}
Identification of flavonoids in Hypericum cordatum (Vell.) N. Robson (Clusiaceae)). The aim of this work was the fractionation of methanolic extracts from stems and leaves of Hypericum cordatum and the identification of flavonoids. The fractionation of methanolic extracts was done using filtration columm in Sephadex LH 20 resin, thin layer and high performance liquid chromatography techniques. Several flavonoids were found in the methanolic extracts, specially flavonols and flavones. Four flavonoids were identified: quercetin, quercitrin, rutin and kaempferol. The identification of these compounds was done by the analyses of the ultraviolet light spectrum obtained in HPLC-UV/DAD and by co-chromatography with standard solutions.
\end{abstract}

Key words - "Cerrado", flavonoids, medicinal plant, phenolic compounds, Saint John's worth

RESUMO - (Identificação de flavonóides em Hypericum cordatum (Vell.) N. Robson (Clusiaceae)). Este trabalho teve como objetivo realizar o fracionamento de extratos metanólicos de caules e folhas de Hypericum cordatum e a identificação de flavonóides. O fracionamento dos extratos foi feito por cromatografia de filtração em resina de Sephadex LH 20, em camada delgada e cromatografia líquida de alta eficiência. Os extratos metanólicos mostraram a presença de vários flavonóides, em especial flavonóis e flavonas. Foram identificados quatro flavonóides: quercetina, quercitrina, rutina e canferol. Os compostos foram identificados pela análise de seus espectros na luz ultravioleta obtidos em CLAE-UV/DAD e por co-cromatografia realizada com os padrões.

Palavras-chave - Cerrado, compostos fenólicos, erva de São João, flavonóides, planta medicinal.

\section{Introdução}

Hypericum cordatum (Vell.) N. Robson é uma espécie de pequeno porte encontrada no Cerrado das regiões Sudeste e Sul do Brasil, que foi selecionada em uma triagem de plantas com atividade antimicrobiana para estudos fitoquímicos (Biota/Fapesp 98/05074-0). Pertence à família Clusiaceae (Guttiferae), que é formada por 49 gêneros, sendo Hypericum o único a ocorrer efetivamente nas regiões temperadas (Robson 1981, Robson 2006).

O gênero Hypericum vem tendo grande destaque na literatura uma vez que várias ações farmacológicas da espécie européia Hypericum perforatum L. vêm sendo confirmadas (Ozturk et al. 1996, Suzuki et al. 1984, Franklin et al. 1999, Barnes et al. 2001, Zou et al. 2004, Silva et al. 2005, Perfumi et al. 2005). Essa espécie, empregada desde a antiguidade por suas atividades medicinais, é atualmente comercializada como fitoterápico no tratamento de depressão média a moderada (Marques

1. Parte da dissertação de Mestrado do primeiro autor, Programa de Pós Graduação em Biodiversidade e Meio Ambiente, Instituto de Botânica de São Paulo.

2. Instituto de Botânica, Seção de Fisiologia e Bioquímica de Plantas, Caixa Postal 3005, 01061-970 São Paulo, SP, Brasil.

3. Autor para correspondência: amladeira@yahoo.com.br
1999). Entre os principais componentes identificados em $H$. perforatum destacam-se como princípios ativos a hiperforina (floroglucinol) e hipericina (antraquinona). Segundo Barnes et al. (2001), parece existir uma interação entre o teor desses compostos e o dos flavonóides encontrados na espécie para que atividade antidepressiva seja observada.

Apesar disso, poucas investigações têm identificado flavonóides nas espécies de Hypericum (Calie et al. 1983, Rocha et al. 1996, Barnes et al. 2001). Flavonóides são compostos comuns na natureza, atuando na atração de polinizadores e como co-pigmentos das antocianidinas. Apresentam diversas atividades biológicas, destacando-se as antiinflamatória, antibiótica, antitumoral e antioxidante (Bruneton 1995). Quimicamente são compostos derivados da via do ácido chiquímico-acetato, caracterizados por apresentarem dois núcleos fenólicos ligados por uma cadeia de três carbonos (Harborne 1984).

No Brasil, algumas espécies brasileiras passaram a ser estudadas. Rocha et al. (1994, 1995, 1996), isolaram floroglucinóis, $\alpha$-pirona, xantonas e ácido betulínico de Hypericum brasiliense Choisy. Abreu et al. (2005) determinaram nessa espécie a distribuição de compostos durante o crescimento da planta, verificando alta concentração de compostos fenólicos na floração e de terpenos na frutificação. Ferraz et al. (2005) verificaram 
benzopiranos com atividade anti-tumoral em Hypericum polyanthemum Klotzsch ex Reichardt. Fenner et al. (2005) verificaram atividade antifúngica em Hypericum ternum A. St.-Hil. e Viana et al. (2005) verificaram atividade anti-depressiva em Hypericum caprifoliatum Cham. \& Schltdl., em função da presença de floroglucinóis que agem no sistema dopaminérgico.

Estudos preliminares realizados com extratos de diferentes partes de Hypericum cordatum indicaram a presença de flavonóides no extrato metanólico, inativo em bioensaios de atividade antifúngica e anti-tumoral (Gallina 1999).

O objetivo do presente trabalho foi realizar o fracionamento dos extratos metanólicos de caules e folhas e a identificação de flavonóides presentes em Hypericum cordatum.

\section{Material e métodos}

Material vegetal - Hypericum cordatum coletado por Cordeiro; material testemunho Cordeiro 1702 depositado no herbário SP.

Coleta - As plantas foram coletadas em Ibiúna (São Paulo, SP) em campo aberto na beira da estrada, nas proximidades da Rodovia SP-250, Km 63, às margens da Rua Caieiras.

Obtenção do pó de folhas e caules - O material foi seco à sombra. Caules e folhas foram separados e pulverizados em moinho Tecnal TE-625.

Preparo do extrato - Os caules e folhas pulverizados foram submetidos à extração seqüencial com solventes de polaridades crescentes (éter de petróleo, clorofórmio e metanol), conforme metodologia descrita por Rocha et al. (1994). Neste trabalho foram analisados apenas extratos metanólicos. Após concentração em rota-evaporador Büchi, obteve-se 59,67 g do extrato de caule e $63,91 \mathrm{~g}$ para o extrato de folha.

Fracionamento dos extratos metanólicos de caule e folha Foi realizado por cromatografia de filtração em coluna $(48,0 \mathrm{~cm}$ x 4,5 cm) de Sephadex LH-20. Utilizou-se como eluente metanol e foram coletadas frações de $50 \mathrm{~mL}$ (Harborne 1984, Rocha et al. 1994). O fracionamento foi monitorado por cromatografia em camada delgada em placa de sílica, desenvolvida em tolueno: acetato de etila: ácido fórmico (5:4:1) sendo as placas posteriormente reveladas com NP-PEG (difenilboriloxietilamina 1,0\% em metanol, seguida de solução de polietilenoglicol 4000 5,0\% em etanol), reagente para detecção de flavonóides (Wagner \& Bladt 2001). As frações que mostraram mobilidade cromatográfica semelhante foram reunidas. As quatro frações que mostraram a presença de flavonóides foram submetidas a novo fracionamento em coluna de Sephadex LH 20, de acordo com a metodologia já descrita.
As novas frações obtidas foram solubilizadas em metanol e submetidas à partição líquido:líquido com tolueno: acetato de etila: ácido fórmico (5:4:1), tendo-se obtido duas fases, a solúvel e a insolúvel nessa mistura de solventes. Tendo em vista a mobilidade das frações solúveis no solvente citado, elas foram submetidas a novos fracionamentos em coluna (40,0 cm x 2,2 cm) de Sephadex LH-20, utilizando-se como eluente metanol. As subfrações obtidas foram monitoradas por cromatografia em camada delgada em placa de sílica no mesmo eluente citado, e reunidas de acordo com a semelhança após revelação com NP-PEG.

As frações insolúveis foram analisadas por cromatografia em camada delgada em cromatoplaca de celulose (Merck) utilizando como eluente butanol: ácido acético: água (3:1:1) e revelação com NP-PEG. Em seguida essas frações insolúveis foram analisadas por eletroforese conforme técnica descrita em Seabra et al. (1991) e Seabra \& Alves (1991). Amostras das frações da fase insolúvel $(10 \mathrm{mg})$ solubilizadas em água ácida com TFA $0,1 \%$ foram submetidas à hidrólise parcial (com $\mathrm{HCl}$ 3,0 N à temperatura ambiente, por 30 minutos) e à hidrólise total (com $\mathrm{HCl} \mathrm{3,0} \mathrm{N} \mathrm{à} 100^{\circ} \mathrm{C}$ por duas horas) $\mathrm{O}$ material hidrolisado foi submetido à partição com acetato de etila e a fração aquosa foi neutralizada com cloreto de bário $1,5 \mathrm{~g}$. A eletroforese foi realizada com as frações insolúveis $(100 \mu \mathrm{g})$ ressuspendidas em $\mathrm{H} 2 \mathrm{O}$ com ácido trifluoroacético (TFA) a $0,1 \%$, que foram aplicadas em papel Whatmann $3 \mathrm{~mm}$, de $20,0 \mathrm{~cm}$ por $35,0 \mathrm{~cm}$. Utilizou-se tampão (buffer) de pH 2,0 com ácido fórmico: ácido acético: água (3,3 mL: 14,7 mL: $182,0 \mathrm{~mL}$ ), e uma tensão de $400 \mathrm{~V}$ por 30, 60 e 90 minutos, em fonte Bio Rad - Power Pac 3000. Após a eletroforese, o papel foi revelado com vapor de amônio para detecção de flavonóides sulfatados.

Posteriormente, as frações solúveis e insolúveis foram analisadas por cromatografia líquida de alta eficiência (CLAE). Para estas análises utilizou-se cromatógrafo Varian equipado com detector na luz ultravioleta (CLAE-UV) e cromatógrafo Dionex equipado com detector de arranjo de diodo (CLAE-UV/DAD). Nas análises nos cromatógrafos Varian e Dionex foram utilizadas colunas analíticas de sílica RP-18, fluxo de $1,0 \mathrm{~mL} \mathrm{~min}^{-1}$. Foram injetados $20,0 \mu \mathrm{L}$ das frações na concentração de $5,0 \mathrm{mg} \mathrm{mL}^{-1}$, e a detecção foi feita a 250 e $370 \mathrm{~nm}$.

Utilizou-se dois gradientes diferentes para eluição dos compostos. $\mathrm{O}$ gradiente de eluição 1 foi programado com rampa variando de 0 a 5 minutos com água $(0,1 \%$ TFA): acetonitrila $(0,1 \%$ TFA) $(95: 5)(\mathrm{V} / \mathrm{V})$, em seguida água (0,1\% TFA): acetonitrila (0,1\% TFA) $(9: 1)(\mathrm{V} / \mathrm{V})$ até 10 minutos, água (0,1\% TFA): acetonitrila $(0,1 \%$ TFA) (8:2) até 20 minutos, água (0,1\% TFA): acetonitrila $(0,1 \%$ TFA) (4:6) (V/V), até 40 minutos, acetonitrila (0,1\% TFA) $100 \%$ até 43 minutos e posteriormente $100 \%$ de metanol até 46 minutos.

O gradiente de eluição 2 foi programado com rampa variando de 0 a 10 minutos com água (0,1\% TFA) $100 \%$, em seguida água ( $0,1 \%$ TFA): acetonitrila $(0,1 \%$ TFA) $(8: 2)$ $(\mathrm{V} / \mathrm{V})$ até 20 minutos, água $(0,1 \% \mathrm{TFA})$ : acetonitrila $(0,1 \%$ 
TFA) (6:4) até 30 minutos, água (0,1\% TFA): acetonitrila $(0,1 \%$ TFA) $(4: 6)(\mathrm{V} / \mathrm{V})$ até 40 minutos, água $(0,1 \% \mathrm{TFA})$ : acetonitrila $(0,1 \%$ TFA) $(2: 8)(\mathrm{V} / \mathrm{V})$ até 50 minutos, acetonitrila (0,1\% TFA) (100\%) até 60 minutos, acetonitrila ( $0,1 \%$ TFA): metanol $(8: 2)(\mathrm{V} / \mathrm{V})$ até 70 minutos e finalmente metanol $100 \%$ até 80 minutos. Utilizou-se acetonitrila e metanol padrão CLAE (J.T. Baker), Ácido triflouracético (Merck) e os padrões rutina, quercitrina, quercetina e canferol, da Sigma.

\section{Resultados e discussão}

O fracionamento por cromatografia em coluna dos extratos metanólicos brutos de caules e de folhas produziu oito frações. Destas, apenas as quatro primeiras mostraram presença de flavonóides e foram selecionadas para análises mais detalhadas (figura 1). As análises das cromatografias em camada delgada das subfrações

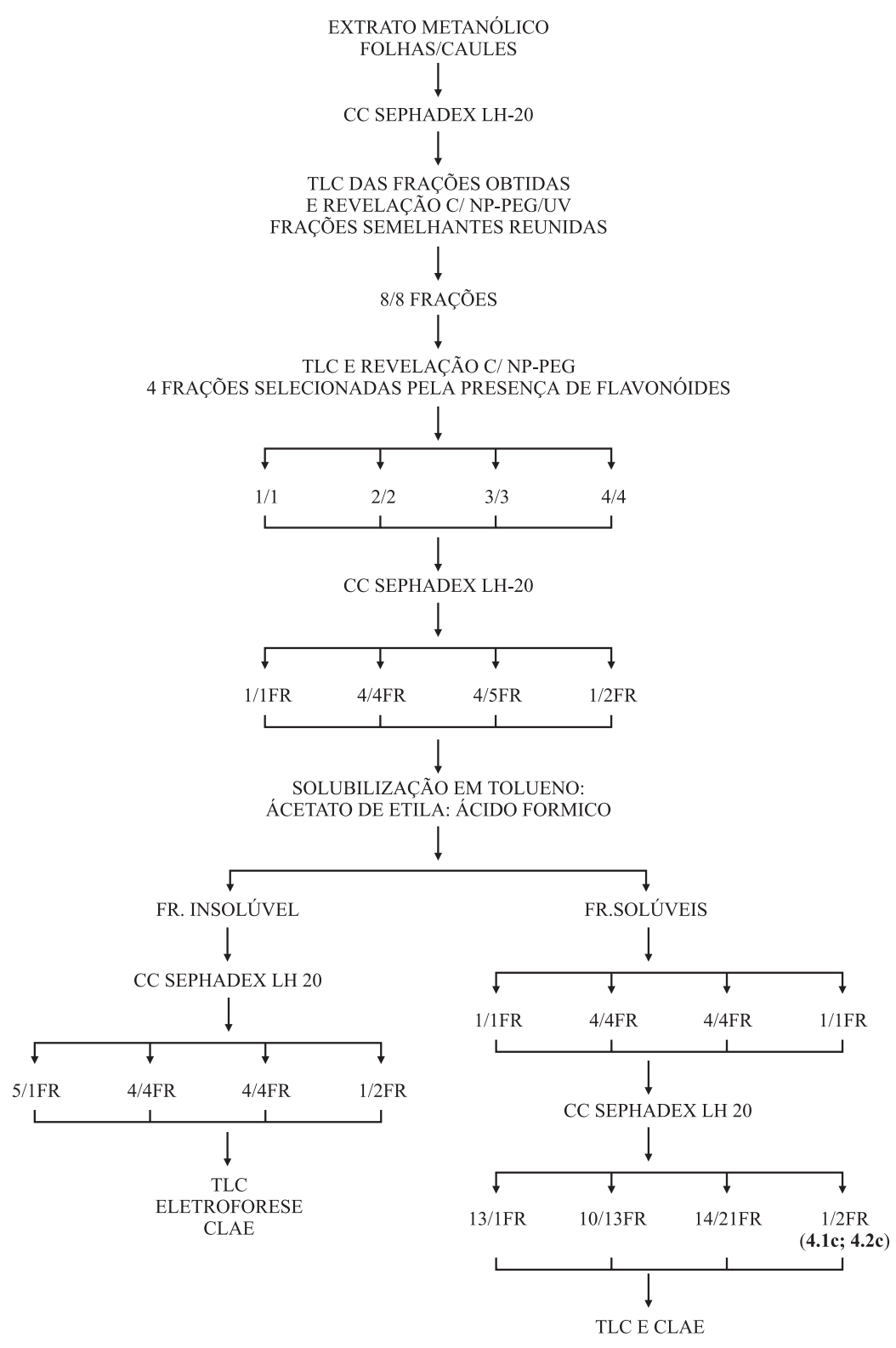

Figura 1. Fracionamentos dos extratos metanólicos de caules e de folhas, com a indicação das frações selecionadas. Legenda: / separa dados de folhas dos de caule. FR - frações, CC - cromatografia em coluna, TLC - cromatografia em camada delgada, NP-PEG - revelador para flavonóides.

Figure 1. Fractionation of metanolic extracts of stems and leaves and the indication of selected fractions. Legend: / separate data of leaves and stems. FR - fractions, CC - column chromatography, TLC - thyn layer chromatography, NP-PEG: reagent for flavonoids detection. 
solúveis obtidas dessas frações mostraram flavonas e flavonóis (Tabelas 1 a 5), fato pouco comum nas espécies de Hypericum, de acordo com Calie et al. (1983). Foram então selecionadas para cromatografia líquida de alta eficiência as frações 4.1 ( $1,8 \mathrm{~g})$ e 4.2 (2,3 g) do extrato de caule, uma vez que mostraram fatores de retenção semelhantes aos de alguns dos padrões de flavonóides, nas análises em cromatografia de camada delgada de sílica.

Inicialmente foram realizadas análises em CLAE-UV da fração 4.1 do extrato de caule, do padrão de canferol, e da fração 4.1 misturada com o padrão (canferol). As

Tabela 1. Rfs dos compostos das frações solúveis 1.1C e 1.1.1F a 1.4.1F. Eluente: Tolueno: Acetato de Etila: Ácido Fórmico (5:4:1). Reagente revelador: NP-PEG, e análise na luz ultravioleta à $250 \mathrm{~nm}$. Glicosídeos de flavonas mostram coloração verde escuro e glicosídeos de flavonol coloração alaranjada ou amarela. O Rf da quercetina foi 0,54 e a cor laranja. $(\mathrm{C}=$ caule, $\mathrm{F}=$ folha $)$.

Table 1. Rfs of the soluble fractions compounds $1.1 \mathrm{C}$ and 1.1.1F - 1.4.1F. Eluent: Toluene: Ethyl acetate: Formic acid (5:4:1). Revelation reagent: NP-PEG, and analysis in UV light $(250 \mathrm{~nm})$. Glucosides of flavones showed green coloration and glucosides of flavonol showed orange or yellow coloration. The $\mathrm{Rf}$ of the standart quercetin was 0,54 with orange color. $(\mathrm{C}=$ stem, $\mathrm{F}=$ leaves $)$.

\begin{tabular}{|c|c|c|}
\hline Frações & $\begin{array}{c}\text { Massa } \\
(\mathrm{mg})\end{array}$ & $\begin{array}{l}\text { Rf - Revelador } \\
\text { NP-PEG UV curto }\end{array}$ \\
\hline $1.1 \mathrm{C}$ & 6,0 & Rastro esverdeado até $\mathrm{Rf} 0,90$ \\
\hline $1.1 .1 \mathrm{~F}$ & 28,6 & $\begin{array}{l}\text { Origem azul; } 0,51 \text { (azul); 0,62 } \\
\text { (verde); 0,78 (verde) }\end{array}$ \\
\hline $1.1 .2 \mathrm{~F}$ & 53,3 & $\begin{array}{l}\text { Origem verde; 0,51 (azul); 0,60 } \\
\text { (verde) }\end{array}$ \\
\hline $1.1 .3 \mathrm{~F}$ & 88,5 & Origem verde \\
\hline $1.1 .4 \mathrm{~F}$ & 13,2 & $\begin{array}{l}\text { Origem escura; 0,71 (escuro); 0,90 } \\
\text { (azul) }\end{array}$ \\
\hline $1.2 .1 \mathrm{~F}$ & 89,7 & Origem verde; 0,50 (verde) \\
\hline $1.2 .2 \mathrm{~F}$ & 12,2 & Origem escura; 0,90 (azul) \\
\hline $1.2 .3 \mathrm{~F}$ & 20,2 & $\begin{array}{l}\text { Origem escura; 0,68 (escuro); 0,75 } \\
\text { (escuro) }\end{array}$ \\
\hline $1.2 .4 \mathrm{~F}$ & 48,9 & Origem verde; 0,57 (verde) \\
\hline $1.2 .5 \mathrm{~F}$ & 48,1 & $\begin{array}{l}\text { Origem escura; 0,11 (marrom- } \\
\text { avermelhado); 0,18 (verde); 0,54 } \\
\text { (amarelo); 0,61 (verde); 0,90 (azul) }\end{array}$ \\
\hline $1.3 .1 \mathrm{~F}$ & 11,9 & $\begin{array}{l}\text { Origem escura; } 0,11 \text { (escuro); } 0,28 \\
\text { (verde); 0,54 (amarelo); 0,90 (azul) }\end{array}$ \\
\hline $1.3 .2 \mathrm{~F}$ & 67,8 & Origem verde \\
\hline $1.4 .1 \mathrm{~F}$ & 4,1 & Origem escura; 0,90 (azul) \\
\hline
\end{tabular}

Tabela 2. Rfs dos compostos da fase solúvel das frações 2.1.1F a 2.4.1F e 2.1.1C a 2.4.3C. Eluente: Tolueno: Acetato de Etila: Ácido Fórmico (5:4:1). Reagente revelador: NP-PEG, e observação na luz ultravioleta à $250 \mathrm{~nm}$. Glicosídeos de flavonas mostram coloração verde escuro e glicosídeos de flavonol coloração alaranjada ou amarela. O Rf da quercetina foi 0,54 e a cor laranja. $(\mathrm{C}=$ caule, $\mathrm{F}=$ folha $)$.

Table 2. Rfs of the soluble fractions compounds $2.1 .1 \mathrm{~F}$ a 2.4.1F e 2.1.1C a 2.4.3C. Eluent: Toluene: Ethyl acetate: Formic acid (5:4:1). Revelation reagent: NP-PEG, and analysis in UV light $(250 \mathrm{~nm})$. Glucosides of flavones showed green coloration and glucosides of flavonol showed orange or yellow coloration. The $\mathrm{Rf}$ of the standart quercetin was 0,54 with orange color. $(\mathrm{C}=$ stem, $\mathrm{F}=$ leaves $)$.

\begin{tabular}{|c|c|c|}
\hline Frações & $\begin{array}{c}\text { Massa } \\
(\mathrm{mg})\end{array}$ & $\begin{array}{l}\mathrm{Rf}-\text { Revelador } \\
\text { NP-PEG UV curto }\end{array}$ \\
\hline $2.1 .1 \mathrm{~F}$ & 12,5 & Origem escura; 0,21 (escuro) \\
\hline $2.1 .2 \mathrm{~F}$ & 13,5 & Origem azul; 0,10 (escuro); 0,52 (escuro) \\
\hline $2.1 .3 \mathrm{~F}$ & 16,9 & $\begin{array}{l}\text { Origem escura; } 0,10 \text { (escuro); 0,52 } \\
\text { (escuro); 0,90 (azul) }\end{array}$ \\
\hline 2.1.4F & 32,4 & Origem escura \\
\hline $2.2 .1 \mathrm{~F}$ & 5,7 & Origem verde; 0,54 (verde); 0,90 (azul) \\
\hline $2.2 .2 \mathrm{~F}$ & 80,4 & $\begin{array}{l}\text { Origem escura; } 0,50 \text { (escura); 0,54 } \\
\text { (alaranjado); 0,71 (verde); 0,90 (azul) }\end{array}$ \\
\hline $2.2 .3 \mathrm{~F}$ & 19,3 & $\begin{array}{l}\text { Origem escura; } 0,20 \text { (verde); } 0,45 \\
\text { (verde); } 0,58 \text { (verde); } 0,90 \text { (azul) }\end{array}$ \\
\hline 2.3.1F & 34,3 & Origem escura \\
\hline $2.3 .2 \mathrm{~F}$ & 17,6 & $\begin{array}{l}\text { Origem escura; 0,11 (marrom- } \\
\text { avermelhado); 0,20 (verde) 0,52 } \\
\text { (escuro); 0,57 (alaranjado); 0,60 (verde) }\end{array}$ \\
\hline 2.4.1F & 16,8 & Origem azul; 0,19 (azul); 0,90 (azul) \\
\hline $2.1 .1 \mathrm{C}$ & 66,5 & $\begin{array}{l}\text { Origem amarela; } 0,14 \text { (verde); 0,50 } \\
\text { (escuro) }\end{array}$ \\
\hline $2.1 .2 \mathrm{C}$ & 124,4 & $\begin{array}{l}\text { Origem escura; } 0,14 \text { (verde); } 0,21 \\
\text { (verde); } 0,42 \text { (verde) }\end{array}$ \\
\hline $2.1 .3 \mathrm{C}$ & 35,6 & Origem escura \\
\hline $2.1 .4 \mathrm{C}$ & 13,1 & Origem escura; 0,50 (escuro); 0,90 (azul) \\
\hline $2.2 .1 \mathrm{C}$ & 21,8 & Origem azul; 0,90 (azul) \\
\hline $2.2 .2 \mathrm{C}$ & 3,7 & Origem escura; 0,14 (escuro) \\
\hline $2.2 .3 \mathrm{C}$ & 91,2 & Origem escura \\
\hline 2.3.1C & 3,9 & Origem escura; 0,90 (azul) \\
\hline 2.3.2C & 115,6 & Origem azul; 0,90 (azul) \\
\hline 2.4.1C & 9,3 & Origem escura; 0,52 (escuro); 0,90 (azul) \\
\hline $2.4 .2 \mathrm{C}$ & 14,8 & $\begin{array}{l}\text { Origem azul; 0,52 (verde); 0,67 (verde); } \\
0,90 \text { (azul) }\end{array}$ \\
\hline $2.4 .3 \mathrm{C}$ & 10,4 & $\begin{array}{l}\text { Origem escura; } 0,45 \text { (esverdeado); 0,90 } \\
\text { (azul) }\end{array}$ \\
\hline
\end{tabular}


Tabela 3. Rfs dos compostos da fase solúvel das frações 3.1.1F a 3.2.4F e 3.1.1C a 3.2.7C. Eluente: Tolueno: Acetato de Etila: Ácido Fórmico (5:4:1). Reagente revelador: NP-PEG, e observação na luz ultravioleta à $250 \mathrm{~nm}$. Glicosídeos de flavonas mostram coloração verde escuro e glicosídeos de flavonol coloração alaranjada ou amarela. O Rf da quercetina foi 0,54 e a cor laranja. ( $\mathrm{C}=$ caule, $\mathrm{F}=$ folha).

Table 3. Rfs of the soluble fractions compounds $3.1 .1 \mathrm{~F}$ a 3.2.4F e 3.1.1C a 3.2.7C. Eluent: Toluene: Ethyl acetate: Formic acid (5:4:1). Revelation reagent: NP-PEG, and analysis in UV light $(250 \mathrm{~nm})$. Glucosides of flavones showed green coloration and glucosides of flavonol showed orange or yellow coloration. The $\mathrm{Rf}$ of the standart quercetin was 0,54 with orange color. $(\mathrm{C}=$ stem, $\mathrm{F}=$ leaves $)$.

\begin{tabular}{|c|c|c|}
\hline Frações & $\begin{array}{c}\text { Massa } \\
(\mathrm{mg})\end{array}$ & $\begin{array}{l}\text { Rf - Revelador } \\
\text { NP-PEG UV curto }\end{array}$ \\
\hline $3.1 .1 \mathrm{~F}$ & 2,0 & Origem clara; 0,85 (azul) \\
\hline $3.1 .2 \mathrm{~F}$ & 23,8 & Origem azul \\
\hline $31.3 \mathrm{~F}$ & 1,6 & $\begin{array}{l}\text { Origem azul; 0,37 (azul); 0,54 (verde); } \\
0,74 \text { (verde); 0,90 (azul) }\end{array}$ \\
\hline $3.1 .1 \mathrm{C}$ & 4,3 & Origem azul; 0,74 (verde); 0,90 (azul) \\
\hline $3.1 .2 \mathrm{C}$ & 2,3 & Origem azul; 0,47 (verde); 0,57 (roxo) \\
\hline $3.1 .3 \mathrm{C}$ & 2,1 & $\begin{array}{l}\text { Origem verde; } 0,57 \text { (roxo); 0,61 (laranja); } \\
0,64 \text { (verde); 0,74 (verde); 0,90 (azul) }\end{array}$ \\
\hline $3.1 .4 \mathrm{C}$ & 1,1 & Origem azul; 0,90 (azul) \\
\hline $3.1 .5 \mathrm{C}$ & 1,8 & Origem azul \\
\hline $3.2 .1 \mathrm{~F}$ & 0,8 & $\begin{array}{l}\text { Origem azul; 0,61 (verde); 0,74 (verde); } \\
0,80 \text { (verde); 0,90 (azul) }\end{array}$ \\
\hline $3.2 .2 \mathrm{~F}$ & 1,3 & $\begin{array}{l}\text { Origem clara; 0,61 (verde); 0,74 (verde); } \\
0,80 \text { (verde); 0,90 (azul) }\end{array}$ \\
\hline $3.2 .3 \mathrm{~F}$ & 0,7 & $\begin{array}{l}\text { Origem clara; 0,61 (verde); 0,74 (verde); } \\
0,80 \text { (verde); 0,90 (azul) }\end{array}$ \\
\hline $3.2 .4 \mathrm{~F}$ & 1,8 & Origem clara \\
\hline $3.2 .1 \mathrm{C}$ & 11,3 & $\begin{array}{l}\text { Origem clara; 0,40 (claro); 0,50 (verde); } \\
0,57 \text { (roxo); 0,61 (laranja); 0,67 (azul); } \\
0,90 \text { (azul) }\end{array}$ \\
\hline $3.2 .2 \mathrm{C}$ & 2,8 & 0,57 (roxo); 0,61 (laranja); 0,67 (azul) \\
\hline $3.2 .3 \mathrm{C}$ & 2,1 & 0,57 (roxo); 0,80 (azul); 0,90 (azul) \\
\hline $3.2 .4 \mathrm{C}$ & 1,8 & Origem clara; 0,25 (verde) \\
\hline $3.2 .5 \mathrm{C}$ & 6,5 & Origem verde; 0,25 (verde); 0,57 (escuro) \\
\hline $3.2 .6 \mathrm{C}$ & 3,0 & Origem clara; 0,61 (verde); 0,74 (verde) \\
\hline $3.2 .7 \mathrm{C}$ & 2,2 & Origem verde; 0,74 (verde) \\
\hline
\end{tabular}

Tabela 4. Rfs dos compostos das frações solúveis 3.3.1F a 3.4.4F e 3.3.1C a 3.4.4C. Eluente: Tolueno: Acetato de Etila: Ácido Fórmico (5:4:1). Reagente revelador: NP-PEG. Observação na luz ultravioleta $250 \mathrm{~nm}$. Glicosídeos de flavonas mostram coloração verde escuro e glicosídeos de flavonol coloração alaranjada ou amarela. O Rf da quercetina foi 0,54 e a cor laranja. $(C=$ caule, $F=$ folha $)$.

Table 4. Rfs of the soluble fractions compounds $3.3 .1 \mathrm{~F}$ a 3.4.4F e 3.3.1C a 3.4.4C. Eluent: Toluene: Ethyl acetate: Formic acid (5:4:1). Revelation reagent: NP-PEG, and analysis in UV light $(250 \mathrm{~nm})$. Glucosides of flavones showed green coloration and glucosides of flavonol showed orange or yellow coloration. The Rf of the standart quercetin was 0,54 with orange color. $(\mathrm{C}=$ stem, $\mathrm{F}=$ leaves $)$.

\begin{tabular}{lcl}
\hline Frações & $\begin{array}{c}\text { Massa } \\
(\mathrm{mg})\end{array}$ & $\begin{array}{l}\text { Rf }- \text { Revelador } \\
\text { NP-PEG UV curto }\end{array}$ \\
\hline $3.3 .1 \mathrm{~F}$ & 1,3 & Origem azul; 0,63 (verde) \\
$3.3 .2 \mathrm{~F}$ & 2,8 & Origem verde; 0,62 (verde) \\
$3.3 .3 \mathrm{~F}$ & 0,9 & Origem azul; 0,55 (verde) \\
$3.3 .4 \mathrm{~F}$ & 1,0 & Origem clara \\
$3.3 .1 \mathrm{C}$ & 6,5 & $\begin{array}{l}\text { Origem verde; } 0,25 \text { (verde); } 0,42 \text { (verde); } \\
\text { 0,52 (verde); } 0,64 \text { (verde); } 0,78 \text { (verde) }\end{array}$ \\
$3.3 .2 \mathrm{C}$ & 3,0 & $\begin{array}{l}\text { Origem verde; } 0,42 \text { (verde); } 0,52 \text { (verde); } \\
0,57 \text { (amarelo); } 0,61 \text { (branco); } 0,78 \text { (verde) }\end{array}$
\end{tabular}

3.3.4C 2,2 Origem verde; 0,42 (verde); 0,52 (verde); 0,57 (amarelo); 0,61 (branco); 0,78 (verde)

3.3.5C 0,8 Origem verde; 0,42 (verde); 0,52 (verde); 0,57 (amarelo); 0,61 (branco); 0,78 (verde)

3.3.6C 5,3 Origem amarela; 0,42 (verde); 0,52 (verde); 0,57 (amarelo); 0,61 (branco); 0,78 (verde)

3.3.7C 3,2 Origem verde

3.4.1F 24,9 Origem verde; 0,40 (verde); 0,50 (azul); 0,57 (verde)

3.4.2F 3,0 Origem verde; 0,48 (verde); 0,61 (amarelo esverdeado); 0,74 (verde); 0,90 (azul)

3.4.3F 1,9 Origem azul; 0,90 (azul)

3.4.4F $\quad 0,8 \quad 0,64$ (verde); 0,74 (verde); 0,90 (azul)

3.4.1C 3,6 Origem verde; 0,74 (verde)

3.4.2C 3,2 Origem verde; 0,60 (verde); 0,74 (verde)

3.4.3C 2,5 Origem amarelada; 0,45 (amarelado); 0,50 (azul); 0,57 (azul); 0,74 (verde)

3.4.4C 1,1 Origem verde; 0,52 (verde amarelado); 0,90 (azul) 
Tabela 5. Rfs dos compostos das frações solúveis 4.1C, 4.2C e 4.1F. Eluente: Tolueno: Acetato de Etila: Ácido Fórmico (5:4:1). Reagente revelador: NP-PEG, e observação na luz ultravioleta $250 \mathrm{~nm}$. Glicosídeos de flavonas mostram coloração verde escuro e glicosídeos de flavonol coloração alaranjada ou amarela. O Rf da quercetina foi 0,54 e a cor laranja. $(\mathrm{C}=$ caule, $\mathrm{F}=$ folha $)$.

Table 5. Rfs of the soluble fractions compounds 4.1C, 4.2C e 4.1F. Eluent: Toluene: Ethyl acetate: Formic acid (5:4:1). Revelation reagent: NP-PEG, and analysis in UV light (250nm). Glucosides of flavones showed green coloration and glucosides of flavonol showed orange or yellow coloration. The $\mathrm{Rf}$ of the standart quercetin was 0,54 with orange color. $(\mathrm{C}=$ stem, $\mathrm{F}=$ leaves $)$.

\begin{tabular}{|c|c|c|}
\hline Frações & $\begin{array}{l}\text { Massa } \\
(\mathrm{mg})\end{array}$ & $\begin{array}{l}\text { Rf - Revelador } \\
\text { NP-PEG UV curto }\end{array}$ \\
\hline $4.1 \mathrm{C}$ & 10,2 & $\begin{array}{l}\text { Origem laranja; } 0,14 \text { (verde); } 0,21 \\
\text { (verde); } 0,28 \text { (verde); 0,41 (verde); } \\
0,54 \text { (laranja) }\end{array}$ \\
\hline $4.2 \mathrm{C}$ & 11,8 & $\begin{array}{l}\text { Origem laranja; } 0,14 \text { (verde); } 0,21 \\
\text { (verde); 0,28 (laranja); 0,38 (verde); } \\
0,41 \text { (verde); 0,54 (laranja); 0,61 } \\
\text { (verde); 0,71 (verde); 0,90 (azul) }\end{array}$ \\
\hline $4.1 \mathrm{~F}$ & 13,2 & $\begin{array}{l}\text { Origem verde; } 0,50 \text { (azul); } 0,54 \\
\text { (verde); 0,61 (verde); } 0,71 \text { (verde); } \\
0,77 \text { (verde); } 0,90 \text { (azul) }\end{array}$ \\
\hline
\end{tabular}

análises foram realizadas em dois gradientes de eluentes para comprovação da identificação do canferol na fração. Houve aumento do pico referente ao composto observado no tempo de retenção 21,4 min. quando a mistura foi analisada com o eluente 1 (figura 2), do mesmo modo que para o pico do composto com tempo de retenção 34,0 min. observado nas análises com o eluente 2 (figura 3). Nas análises realizadas em CLAE-UV/DAD foi possível obter os espectros na região do ultravioleta para o composto com tempo de retenção $24,1 \mathrm{~min}$. na fração 4.1 e para o canferol, comprovando assim esse dado (figura 4). Pode-se então concluir que canferol ocorre em Hypericum cordatum.

As figuras 5 e 6 apresentam os perfis cromatográficos obtidos nas análises em CLAE-UV da fração 4.2 do extrato de caule, dos padrões de flavonóides (rutina, quercitrina e quercetina) e da mistura da fração $4.2 \mathrm{com}$ padrões de flavonóides. Verificou-se aumento do pico dos compostos com tempo de retenção 17,1; 18,2 e 21,4 min. para o eluente 1 e do pico dos compostos com tempos de retenção 25,9; 27,4 e 31,0 min. para o eluente 2 . As

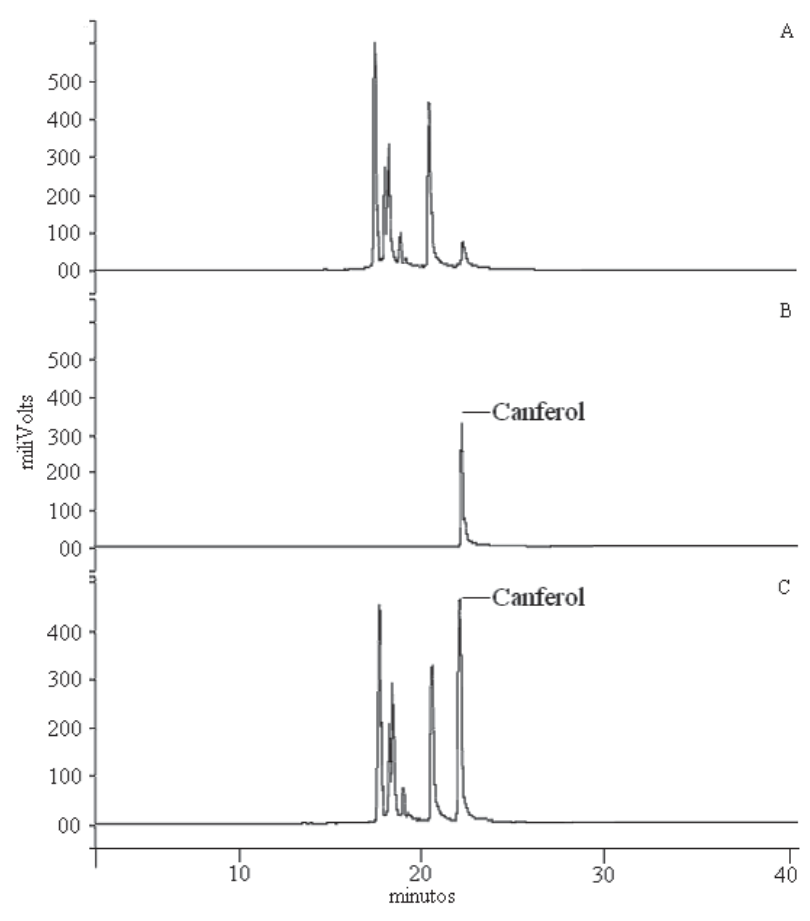

Figura 2. Perfil cromatográfico da fração 4.1 da fase insolúvel do extrato metanólico de caule $\left(5 \mathrm{mg} \mathrm{mL}^{-1}\right)(\mathrm{A})$; do padrão de canferol $\left(0,5 \mathrm{mg} \mathrm{mL}^{-1}\right)$ (B) e da mistura da fração 4.1 com o padrão de canferol (solução diluída 1:1 $\mathrm{V}: \mathrm{V})(\mathrm{C})$. Análise em CLAE-UV realizada com o eluente 1. Detecção a $370 \mathrm{~nm}$.

Figure 2. Chromatographic profile of fraction 4.1 of insoluble phase of methanolic extract of stem $\left(5 \mathrm{mg} \mathrm{mL} \mathrm{m}^{-1}\right)$ (A); kaempferol standard (B) and fraction 4.1 with the kaempferol standard (C). Analysis in HPLC-UVwith elution gradient 1 . Detection at $370 \mathrm{~nm}$.

figuras 7, 8 e 9 apresentam os espectros na região do ultravioleta dos compostos com tempos de retenção 17,1 min., 18,2 min. e 21,4 min. da fração 4.2 que são semelhantes aos espectros dos padrões de flavonóides rutina, quercitrina e quercetina, respectivamente. A presença de quercetina, quercitrina e rutina, foi também identificada nas frações solúveis 1.3.1 (88,5 mg) e 2.2.3 $(19,3 \mathrm{mg})$ do extrato metanólico de folhas (dados não apresentados).

Quercetina e canferol são flavonóides comuns em vegetais. Rutina e quercitrina são flavonóides glicosilados encontrados em um menor número de espécies. Esses quatro flavonóides foram identificados em $\mathrm{H}$. perforatum, que também contém luteolina, hiperosídeo, isoquercitrina, os biflavonóides biapigenina e amentoflavona além de flavonóides associados a taninos condensados como a catequina (Marques 1999, Barnes et al. 2001). A espécie Hypericum brasiliense Choisy possui os mesmos quatro 


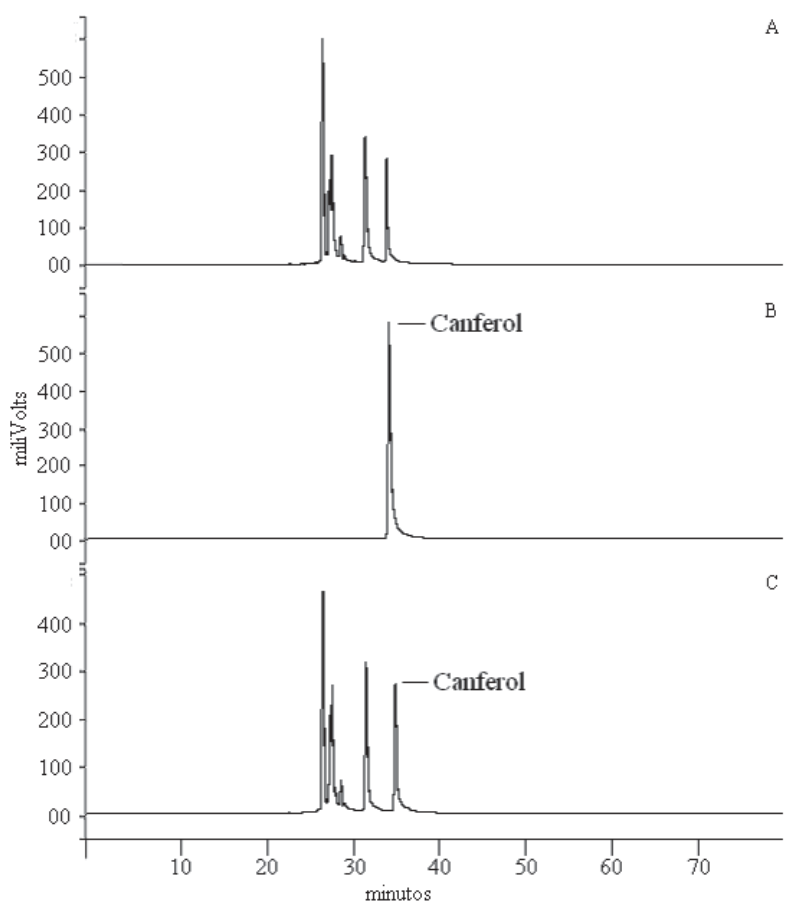

Figura 3. Perfil cromatográfico da fração 4.1 da fase insolúvel do extrato metanólico de caule $\left(5 \mathrm{mg} \mathrm{mL}^{-1}\right)(\mathrm{A})$; do padrão de canferol $\left(0,5 \mathrm{mg} \mathrm{mL}^{-1}\right)(\mathrm{B})$ e da mistura da fração 4.1 com o padrão de canferol (solução diluída 1:1 $\mathrm{V}: \mathrm{V})(\mathrm{C})$. Análise em CLAE-UV realizada com o eluente 2. Detecção a $370 \mathrm{~nm}$.

Figure 3. Chromatographic profile fraction of 4.1 of insoluble phase of methanolic stem $\left(5 \mathrm{mg} \mathrm{mL}^{-1}\right)(\mathrm{A})$; kaempferol standard (B) and fraction 4.1 with the kaempferol standard (C). Analysis in HPLC-UVwith elution gradient 2. Detection at $370 \mathrm{~nm}$.

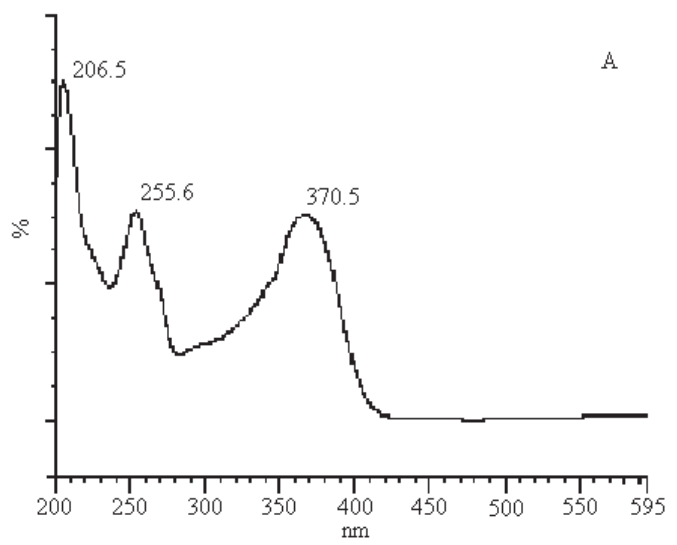

flavonóides identificados em Hypericum cordatum, e compostos fenólicos ocorrem em maior concentração na época da floração (Rocha et al. 1995, Abreu et al. 2005).

As sub-frações insolúveis não saíram da origem das placas de sílica, quando o solvente empregado foi tolueno: acetato de etila: ácido fórmico (5:4:1) e foram arrastadas para o Rf 0.50 nas análises cromatográficas realizadas em butanol: ácido acético: água (3:1:1). Assim sendo cogitou-se a possibilidade de se tratarem de flavonóides sulfatados como a quercetina 3 glucuronide-3'sulfato e a quercetina 3 sulfato, identificadas em três espécies de Hypericum (Seabra \& Alves 1991, Seabra et al., 1991). A realização de eletroforese com essas sub-frações insolúveis mostrou que não há compostos sulfatados nessa espécie.

Apesar dos flavonóides apresentarem atividades antimicrobianas e de ter sido comprovada sua ocorrência nos extratos metanólicos de Hypericum cordatum, atividades anti-fúngicas e antibacterianas não foram produzidas por eles (Gallina 1999).

Além disso atividade antidepressiva não foi verificada em estudos farmacológicos feitos com essa espécie e com Hypericum brasiliense (Mendes et al. 2002). Em Hypericum perforatum hiperosídeo e hipericina mostraram atividade anti-depressiva (Luo et al. 2004), e em Hypericum caprifoliatum a atividade anti-depressiva está relacionada a um floroglucinol (Viana et al. 2005). Por outro lado, hipericina não foi detectada em nenhuma espécie brasileira (Abreu et al. 2005, Fenner et al. 2005, Viana et al. 2005). Assim sendo, parece que realmente há necessidade de uma interação entre vários compostos para que atividade anti-depressiva seja produzida por espécies de Hypericum.

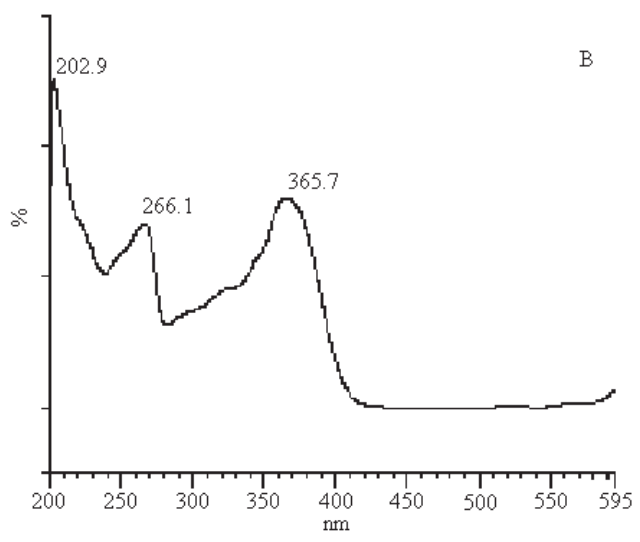

Figura 4. Espectro na região do ultravioleta do composto de tempo de retenção 24,1 minutos da fração 4.1 da fase insolúvel do extrato metanólico de caule (A) e do padrão de canferol (B), obtidos nas análises em CLAE-DAD realizadas com o gradiente da Tabela 1.

Figure 4. UV spectrum of the compound with retention time 24.1 minutes of fraction 4.1 of insoluble phase of methanolic extract of stems $\left(5 \mathrm{mg} \mathrm{mL}^{-1}\right)(\mathrm{A})$ and kaempferol standard (B), obtained in HPLC-UV/DAD analysis with elution gradient 1. 


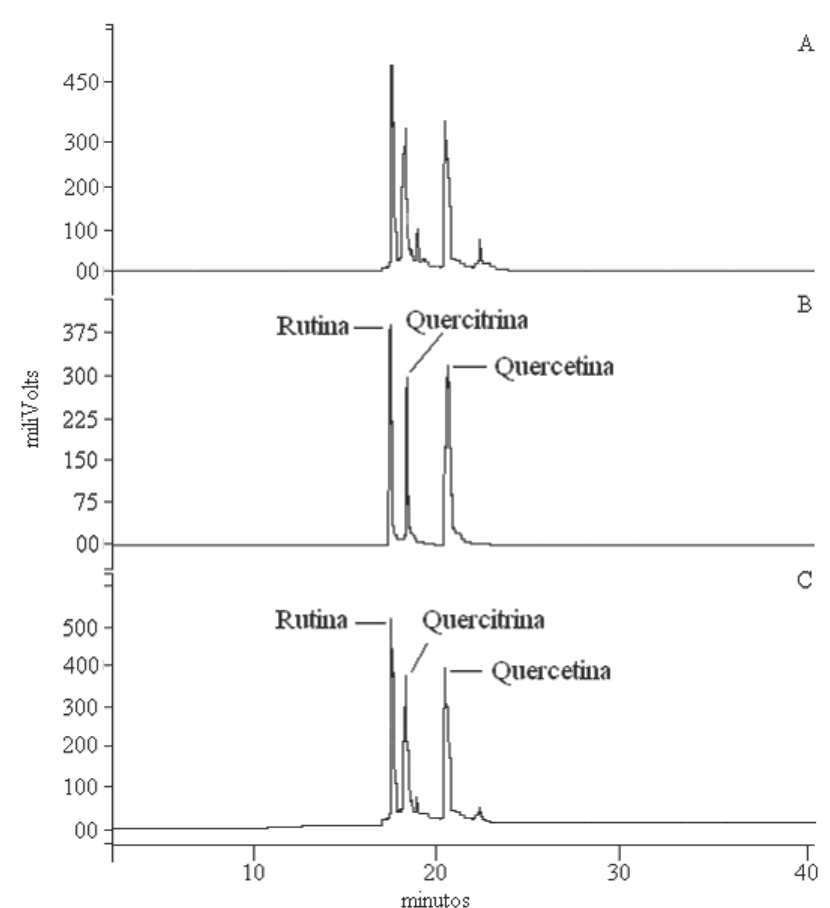

Figura 5. Perfil cromatográfico da fração 4.2 da fase insolúvel do extrato metanólico de caule $\left(5 \mathrm{mg} \mathrm{mL}^{-1}\right)$ (A), dos padrões de flavonóides (rutina, quercitrina e quercetina) $\left(0,5 \mathrm{mg} \mathrm{mL}^{-1}\right)$ (B) e da mistura da fração 4.2 com os padrões de flavonóides (solução diluída 1:1 - V:V) (C). Análise em CLAE-UV realizada com eluente 1 . Detecção a $370 \mathrm{~nm}$.

Figure 5. Chromatographic profile of fraction 4.2 of insoluble phase of methanolic stem extract $\left(5 \mathrm{mg} \mathrm{mL}^{-1}\right)(\mathrm{A})$, flavonoids (rutin, quercitrin and quercetin) $\left(0,5 \mathrm{mg} \mathrm{mL}^{-1}\right)(\mathrm{B})$ and the fraction 4.2 with the flavonoids standards (dilution of the solution 1:1 - V:V) (C). Analysis in HPLC-UV with elution gradient 1 . Detection at $370 \mathrm{~nm}$.

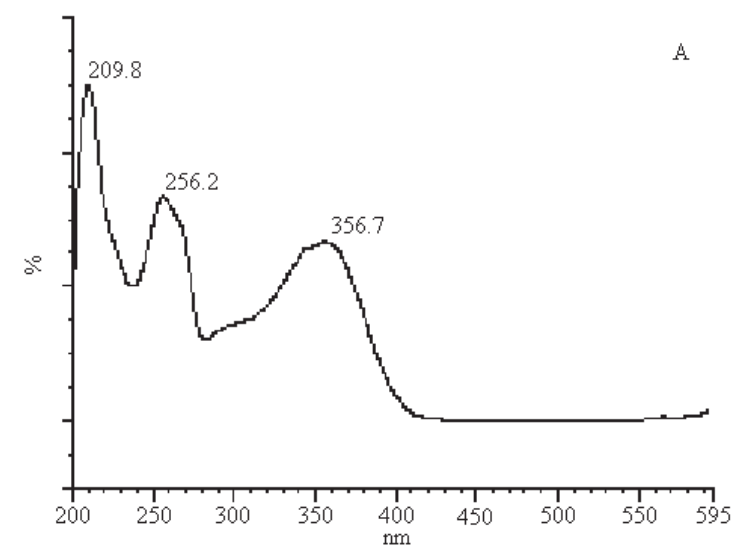

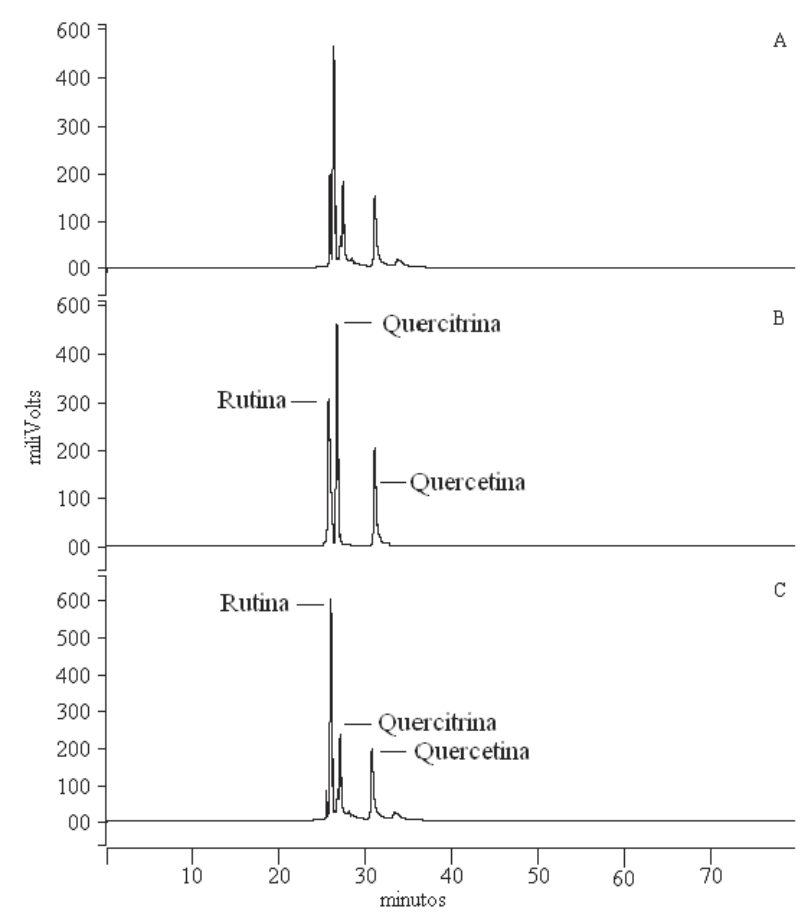

Figura 6. Perfil cromatográfico da fração 4.2 da fase insolúvel do extrato metanólico de caule $\left(5 \mathrm{mg} \mathrm{mL}^{-1}\right)(\mathrm{A})$, dos padrões de flavonóides (rutina, quercitrina e quercetina) $\left(0,5 \mathrm{mg} \mathrm{mL}^{-1}\right)$ (B) e da mistura da fração 4.2 com os padrões de flavonóides (solução diluída 1:1 - V:V) (C). Análise em CLAE-UV realizada com eluente 2. Detecção a $370 \mathrm{~nm}$.

Figure 6. Chromatographic profile of fraction 4.2 of insoluble phase of methanolic stem extract $\left(5 \mathrm{mg} \mathrm{mL}^{-1}\right)(\mathrm{A})$, flavonoids (rutin, quercitrin and quercetin) $\left(0,5 \mathrm{mg} \mathrm{mL}^{-1}\right)$ (B) and fraction 4.2 with the flavonoids standards (dilution of the solution $1: 1-\mathrm{V}: \mathrm{V})(\mathrm{C})$. Analysis in HPLC-UV with elution gradient 2. Detection at $370 \mathrm{~nm}$.

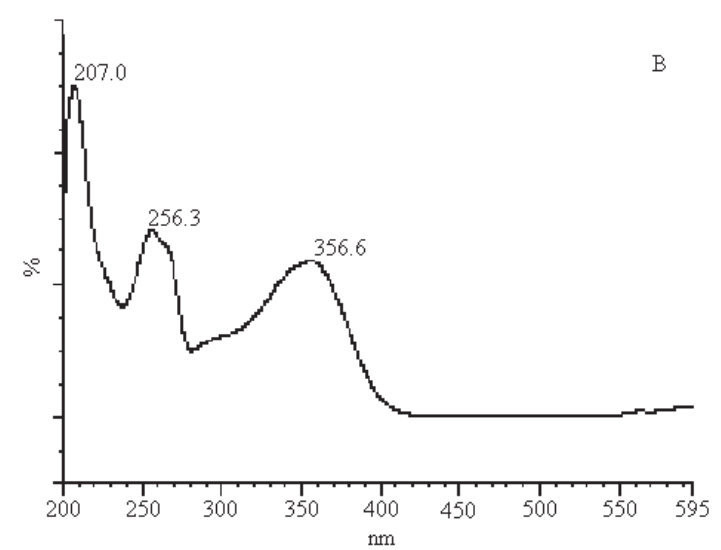

Figura 7. Espectro na região do ultravioleta do composto com tempo de retenção 17,1 minutos da fração 4.2 da fase insolúvel do extrato metanólico de caule (A) e do padrão de rutina (B), obtidos em CLAE-DAD realizadas com o eluente 1.

Figure 7. UV spectrum of the compound with retention time 17.1 minutes of fraction 4.2 of insoluble phase of methanolic extract of stems (A) and rutin standard (B), obtained in HPLC-UV/DAD analysis with elution gradient 1. 

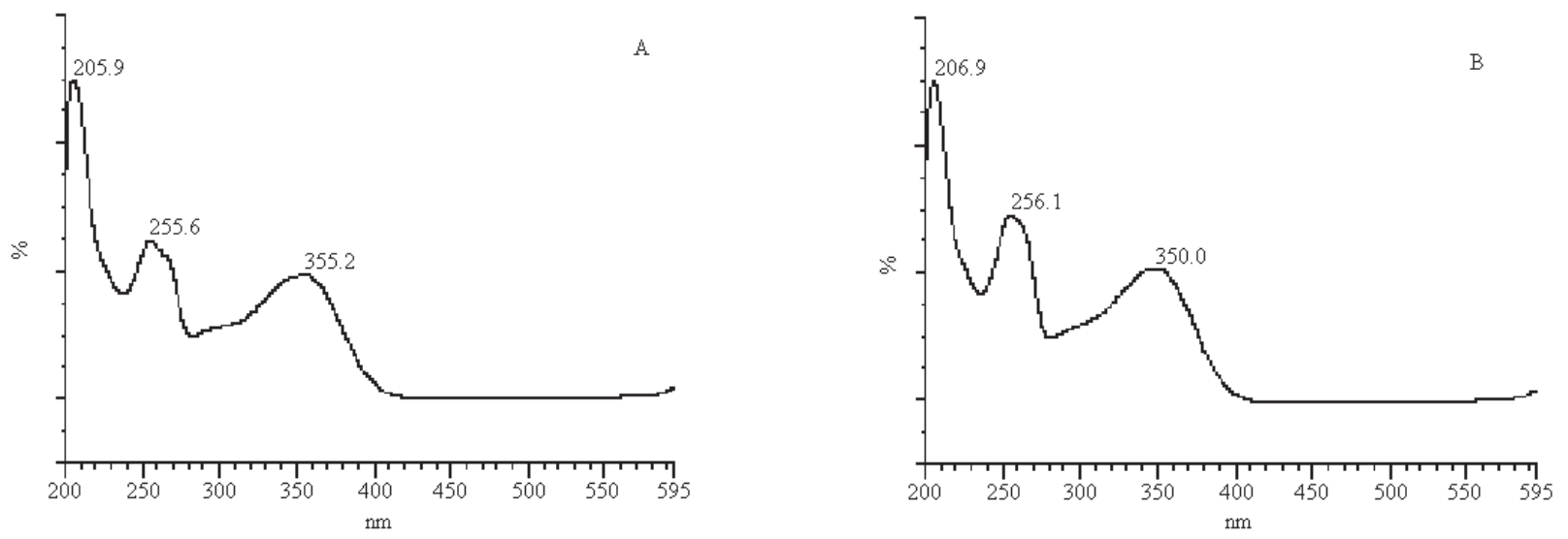

Figura 8. Espectro na região do ultravioleta do composto com tempo de retenção 18,2 minutos da fração 4.2 da fase insolúvel do extrato metanólico de caule (A) e do padrão de quercitrina (B), obtidos em CLAE-DAD realizadas com o eluente 1.

Figure 8. UV spectrum of the compound with retention time 18.2 minutes of fraction 4.2 of insoluble phase of methanolic extract of stems (A) and quercitrin standard (B), obtained in HPLC-UV/DAD analysis with elution gradient 1.
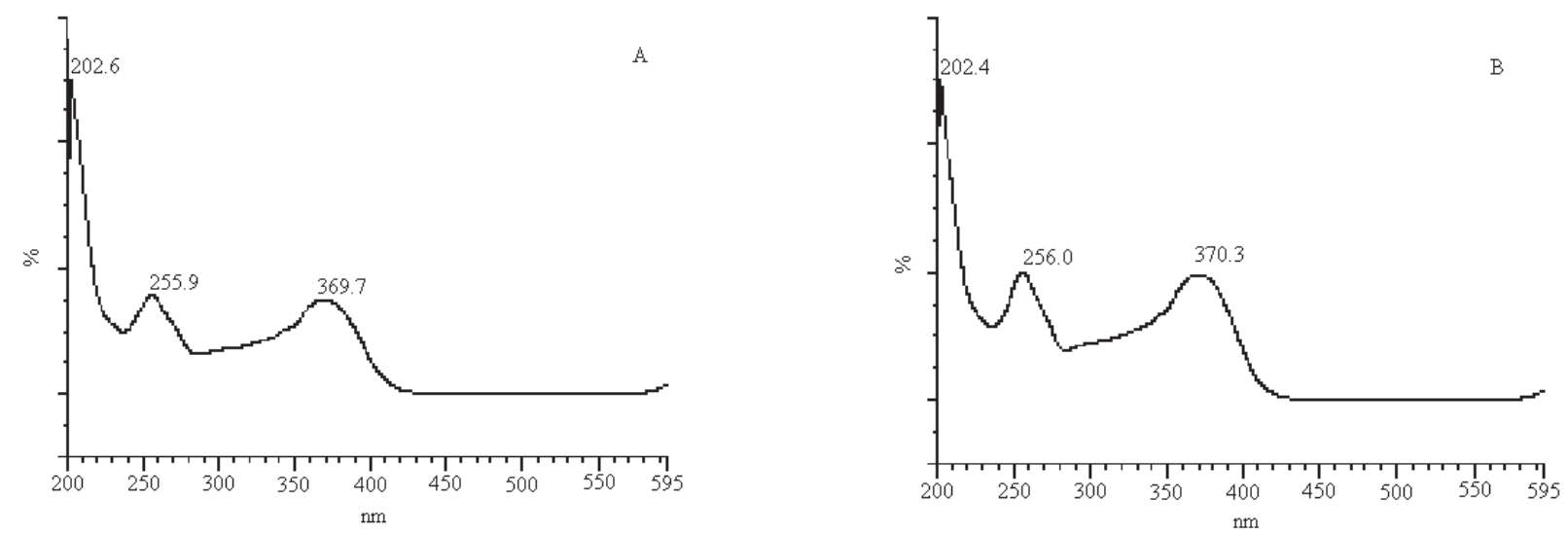

Figura 9. Espectro na região do ultravioleta do composto com tempo de retenção 21,4 minutos da fração 4.2 da fase insolúvel do extrato metanólico de caule (A) e do padrão de quercetina (B), obtidos em CLAE-DAD realizadas com o eluente 1.

Figure 9. UV spectrum of the compound with retention time 21.4 minutes of fraction 4.2 of insoluble phase of methanolic extract of stems (A) and quercetin standard (B), obtained in HPLC-UV/DAD analysis with elution gradient 1.

No presente trabalho foi obtida a identificação parcial de quatro flavonóides descritos também em outras espécies do gênero. A identificação de outros flavonóides detectados dependerá de novas etapas de purificação dos componentes das frações obtidas, que serão objeto de estudos futuros.

Agradecimentos - Os autores agradecem à Fapesp pelos auxílios concedidos (98/05074-0 e 00/05761-0), à Dra. Luciana Retz de Carvalho pela permissão de utilização do equipamento CLAE-UV/DAD, pertencente à Seção de Ficologia do Instituto de Botânica, e às Dras. Maria Claudia Marx Young e Luce Maria Brandão Torres pelas sugestões dadas durante a realização deste trabalho.

\section{Referências Bibliográficas}

ABREU, I.N., PORTO, A.L.M., MARSAIOLI, A.J. \& MAZZAFERA, P. 2005. Bioactive substances in Hypericum brasiliense Choisy during plant growth. Plant Science 167:949-954.

BARNES, J., ANDERSON, L.A. \& PHILLIPSON, J.D. 2001. St John's worth (Hypericum perforatum). A review of chemistry, pharmacology and clinical properties. Journal of Pharmacy and Pharmacology 53:583-600.

BRUNETON, J. 1995. Phenols and phenolic acids In Pharmacognosy, phytochemistry and medical plants (J. Bruneton, ed.). Lavoisier Press, EUA, p.211-227. 
CALIE, P.J., SCHILLINGS, E.E. \& WEBB, D.H. 1983. Flavonoids of the segregates Ascyron and Cookea from Hypericum. Biochemical Systematic and Biology 2:107-109.

FENNER, R., SORTINO, M., KUZE RATES, S.M., DALL'AGNOLL, R., FERRAZ, A., BERNARDI, A.P., ALBRIG, D., NOR, C., von POSER, G., SCHAPOVAL, E. \& ZACHINO, S. 2005. Antifungal activity of some Brazilian Hypericum species. Phytomedicine 5:213-217.

FERRAZ, A.B.F., GRIVICICH, I., von POSER, G.L., FARIA, D.H., KAISER, G.B., SCHWARTSMANN, G., HENRIQUES, A.T. \& ROCHA, A.B. 2005. Antitumour activity of three benzopirans isolated from Hypericum polyanthenum. Fitoterapia 216:210-215.

FRANKLIN, M., CHI, J., MCGAWAIN, C., HOCKNEY, R, REED, A., CAMPLING, G.; WALE, R.W., \& COWAN, P.J. 1999. Neuroendocrine evidence for dopaminergic actions of Hypericum extracts (LI 60) in health volunteers. Biological Psychiatry 46:581-584.

GALLINA, P. 1999. Análise de compostos secundários de Hypericum cordatum (Vell. Conc.) N. Robson, comb. Nov (Gutiferae). Monografia de graduação. Universidade Metodista, São Paulo.

HARBORNE, J.B. 1984. Phytochemical Methods. A guide to modern techniques of plant analysis. Chappman \& Hall. Londres.

LUO, L., SUN, Q., MAO, Y.Y., LU, Y.H. \& TANH, R.X. 2004. Inhibitory effects of flavonoids from Hypericum perforatum on nitric oxide synthase. Ethnopharmacology 93:221-225.

MARQUES, L.C. 1999. Hypericum e kava-kava: Aspectos farmacológicos e terapêuticos. Revista Racine 51:56-62.

MENDES, F.R., MATTEI, L. \& CARLINI, E.L.A. 2002. Activity of Hypericum brasiliense and Hypericum cordatum on the central nervous system in rodents. Fitoterapia 73:462-471.

OZTURK, Y., AYDIN, S., BEIS, R., BASER, K.H.C. \& BERBEROGLU, H. 1996. Effects of Hypericum perforatum and Hypericum calycinum L. extract on central nervous system in mice. Phytomedicine 3:139-146.

PERFUMI, M., MATTIOLI, L., CUCCUELLI, M. \& MASSI, M. 2005. Reduction of ethanol intake by chronic treatment with Hypericum perforatum, alone and combined with naltrexone in rats. Journal of Psychopharmacology 19:448-454.
ROBSON, N.K.B. 1981. Studies in genus Hypericum L. (Gutiferae) 2. characters of genus. Bulletin of the British Museum (Natural History), Botany 8:55-226.

ROBSON, N.K.B. 2006. Studies in genus Hypericum L. (Clusiaceae). Section 9 Hypericum sensu lato (part 3): Subsection 1. Hypericum series 2. Senanensia, subsection 2. Erecta and section 9b. Graveolentia. Systematic and Biodiversity 4:19-98.

ROCHA, L., MARTSON, A., KAPLAN, M.A.C., EVANS, H.S., TULL, U., TESTA, B. \& HOSTETTMANN, K. 1994. An antifungal $\gamma$-pyrone and xanthones with monoaminoxidase inhibitory activity from Hypericum brasiliense. Phytochemistry 36:1381-1385.

ROCHA, L., MARTSON, A., POTTERAT, O., KAPLAN, M.A.C. \& HOSTETTMANN, K. 1995. Antibacterial phloroglucinol and flavonoids from Hypericum brasiliense. Phytochemistry 40:1447-1452.

ROCHA, L., MARTSON, A., POTTERAT, O., KAPLAN, M.A.C., EVANS, H.S. \& HOSTETTMANN, K. 1996. More phloroglucinols from Hypericum brasiliense. Phytochemistry 42:185-188.

SEABRA, R.M. \& ALVES, A.C. 1991. Quercetin-3-sulphate from Hypericum elodes. Phytochemistry 30:1344-1345.

SEABRA, R.M., VASCONCELOS, M.H. \& ALVES, A.C. 1991. Flavonoids sulphates from Hypericum undulatum. Revista Portuguesa de Farmacologia 36:16-18.

SILVA, B.A., FERRERES, F., MALVA, J.O. \& DIAS, A.C.P. 2005. Phytochemical and antioxidant caracterization of Hypericum perforatum alcoholic extracts. Food Chemistry 90:157-167.

SUZUKI, O., KATSUMATA, Y., OYS, M., BLADT, S. \& WAGNER, H. 1984. Inhibition of monoaminooxidase by Hypericum. Planta Medica 50:272-274.

VIANA, A., REGO, J.C., von POSER, G., FERRAZ, A., HECKLER, A.P., COSTENTINI, J. \& RATES, S.M.K. 2005. The antidepressant effect of Hypericum caprifoliatum Cham \& Schlecht (Guttiferae) on forced swimming test result from an inhibition of neuronal monoamino uptake. Neuropharmacology 49:1042-1052.

WAGNER, H. \& BLADT, S. 2001. Plant drug analysis. Thin Layer Chromatography Atlas. $2^{\text {nd }}$ ed. Springer. Londres.

ZOU, Y., LU, Y. \& WEI, D. 2004. Hypocholesterolemic effects of a flavonoid rich extract of Hypericum perforatum L. in rats fed a cholesterol rich diet. Journal of Agricultural and Food Chemistry 53:2462-2466. 